\title{
An Investigation of Dopamine's Role in Six Psychiatric Illnesses
}

\author{
Alyssa Wang ${ }^{1}$ and Julian Day-Cooney ${ }^{1}$ \\ ${ }^{1}$ Horizon Academic Research Program, Jersey City, NJ, USA
}

\section{ABSTRACT}

This paper aims to investigate the neurotransmitter dopamine's role in six prevalent psychiatric disorders: attentiondeficit disorder, autism spectrum disorder, bipolar disorder, major depression, post-traumatic stress disorder, and schizophrenia. Although dopamine's contribution to schizophrenia is the most understood, dopamine's role in other disorders is also an increasing area of research. Dopamine affects reward-motivated behavior, motor control, cognition, and possesses many other sub-functions as well. Dysfunction in dopamine firing rate, firing time, tonic and phasic levels, and receptor and transporter density can lead to numerous diseases and symptoms. Having a better understanding of how dopamine affects these illnesses specifically can lead to the development of more effective treatment and medication. Thus, the analysis of the studies and proposals for how dopamine is involved in various mental disorders are discussed in this review.

\section{Introduction}

Dopamine is a neurotransmitter that plays an important role in movement, pleasure, reward, behavior, cognition, attention, memory, sleep, mood, and learning in humans and animals. Dopamine is synthesized and secreted by dopaminergic neurons of the ventral tegmental area (VTA), the substantia nigra, and the hypothalamus. The neurotransmitter's excess or deficiency can result in movement disorders, drug addiction, and psychiatric illnesses (Mandal, 2019). Excess in dopamine levels typically causes the body to make unnecessary movements whereas decreased dopamine leads to delayed and uncoordinated movements. A prevalent movement disorder involving a severe drop in dopamine levels is Parkinson's disease: the most notable symptoms include tremors, stiffness, and impaired balance and coordination (NIH, 2017). Furthermore, drugs that inhibit the reuptake of dopamine such as cocaine and amphetamines can cause serious addiction issues. Cocaine blocks dopamine transporters, increasing dopamine presence in the synaptic cleft. Amphetamines perform the same function, but due to their structure being similar to dopamine, they can enter the presynaptic neuron through its dopamine transporters. Thus, the drug forces dopamine molecules out of storage vesicles, leading to feelings of pleasure and addiction.

Dopamine also affects pleasure in reward-seeking behavior, memory, attention, cognition, and problem-solving (Mandal, 2019). Because dopamine has this variety of functions, abnormalities involving dopamine levels, dopamine firing rates, dopamine receptor efficiency, and neurotransmission may lead to many different consequences. Hindered capability in memory, attention, and perceiving reward directly correspond to symptoms in attention deficit hyperactivity disorder (ADHD). Dopamine dysfunction also encompasses the inability to perceive normally rewarding social interactions as rewarding and constant, stereotypic movement, which are symptoms in autism spectrum disorder. Since decreased dopamine levels may result in a lack of feeling of reward and motivation, the neurotransmitter may also be involved in major depressive disorder. The dopamine hypothesis of psychosis (also known as the dopamine hypothesis of schizophrenia) argues that hyperactivity of dopamine D2 receptor neurotransmission leads to positive symptoms in schizophrenia such as hallucinations and delusions, which in turn, are also common symptoms in patients with bipolar disorder with psychosis. Additionally, since dopamine affects fear and anxiety, hyperactivity 
relating to dopamine may trigger constant stress responses in those with post- traumatic stress disorder (PTSD). Though research dedicated specifically to dopamine's role in these psychiatric illnesses is limited, the neurotransmitter is becoming an increased area of investigation due to its many different functions. A better understanding of dopamine is crucial in finding more effective forms of medication. So, this review intends to prove that the neurotransmitter dopamine is involved in the six psychiatric illnesses of ADHD, autism, bipolar disorder, depression, PTSD, and Schizophrenia.

\section{Discussion}

\section{Attention Deficit Hyperactivity Disorder}

Attention deficit hyperactivity disorder's (ADHD) most prevalent symptoms include hyperactivity, inattention, impulsivity, forgetfulness, fidgeting, and aggression. Due to the wide range of negative effects ADHD has upon the population, such as these symptoms and increased financial burdens among families and societies, this disorder is a serious public health issue (Polanczyk et al., 2007). Though different studies conclude drastically different rates of this disorder across the globe - from $1 \%$ to as high as $20 \%$ of children in the population - ADHD remains one of the most largely researched mental illnesses (Polanczyk et al., 2007). ADHD is significantly more prevalent in the United States as compared to other countries, causing a medical debate about the influence of cultural factors on the disorder. Nevertheless, having a greater understanding of the causes of this hyperactivity in the brain is crucial for future treatments and medications. Research is emerging that dopamine may contribute to some of the symptoms of ADHD.

Dopamine has been implicated in causing ADHD through reward and motivation. A reward is defined as any object or goal that is sought after through the allocation of time, effort, or energy. Rewards are important for individual support such as eating, drinking, and reproduction, but also non-alimentary and nonsexual activities such as gambling. It is proposed that one neural system mainly processes reward, and it is supported that the mesencephalic dopamine system contributes to the response to rewards (Arias-Carrián et al., 2010). The benefits of positive reinforcement on developing cognitive skills are more significant for children with ADHD. Additionally, people with ADHD have a tendency to show favoritism towards short-term and smaller rewards rather than looking towards long-term favorable outcomes. So, dopamine system dysfunction likely affects motivation and reward processing in those with ADHD.

The dopamine system is proved to be relevant to reward because of the animal work analyzed by Schultz which depicted that the firing rate of dopamine neurons in both the ventral tegmental area (VTA) and the substantia nigra increases in response to an unpredicted reward and decreases in response to a predicted reward being withheld (Schultz et al., 1997). Through studying reward in monkeys and rats, the results indicate that dopamine response encodes for expectations about stimuli or reward. Dopamine neurons not only report the occurrence of reward but also codes for a deviation between the actual reward and predictions of that reward. These neurons are only activated if the reward is still uncertain and are excellent detectors of the "goodness" of the learned prediction of the event. Dopamine neurons produce an increased spike of production if an event is better than predicted, no change in spike production if the event occurs as predicted, and a decreased spike in production if the event is worse than predicted (Schultz et al., 1997).

There are two theories proposing that ADHD correlates with the dysfunction of the midbrain dopamine system. First, the dynamic developmental theory (DDT) explains how dopamine causes ADHD and proposes that dopamine is accountable for the more abrupt delay-of-reinforcement gradient, resulting in a shorter dopamine cell firing time (a neuron emitting an action potential, or a nerve impulse is commonly known as "firing") (Luman et al., 2010). Additionally, a steeper gradient of dopamine firing means that a stimulus that is positively-reinforcing loses value quickly when there is a delay imposed, which leads to impulsive behavior (Sagvolden et al., 2005). This gradient is caused by decreased amounts of tonic dopamine. Tonic activity involves spontaneously firing dopamine neurons which often has the tendency to fire slowly and erratically. In contrast, phasic activity is when dopamine neurons fire in quick bursts of larger action potentials. Lower tonic dopamine levels also caused dips in phasic dopamine after the 
withholding of the reward, so children with ADHD would exhibit slower extinction of their behavior (Sagvolden et al., 2005).

Second, the dopamine transfer deficit theory (DTD) proposes altered activity in phasic dopamine response to reinforcement as the explanation of altered reinforcement sensitivity whereas the DDT assumes low levels of tonic dopamine (Luman et al., 2010). DTD estimates abnormalities in the firing of the dopamine cells in response to cues that predict reinforcement (figure 1). In healthy individuals, when a reward is repeatedly administered with a predictor (being the aforementioned cue), dopamine neurons start firing to the predictor itself and the reward. Once the reward to predictor relationship is clear, dopamine fires only to the predictor (figure 1). Dopamine cell firing is abnormal in patients with ADHD. Even when time passes and the cue of the reward is supposed to be learned over time, those with ADHD still express a much higher firing rate after the actual reward as opposed to the cue itself.

Children with ADHD show issues with anticipating rewards and controlling behavior. Less efficient learning is also associated with delayed response to immediate reward. Weaker conditioning to reward results in faster extinction of behavior and weaker impact of reinforcers on behavior over increased increments of time (Tripp \& Wickens, 2008). Although the DTD and the DDT propose different explanations for the cause of dopamine dysfunction, both concurthat dopamine cell firing is relevant to ADHD.

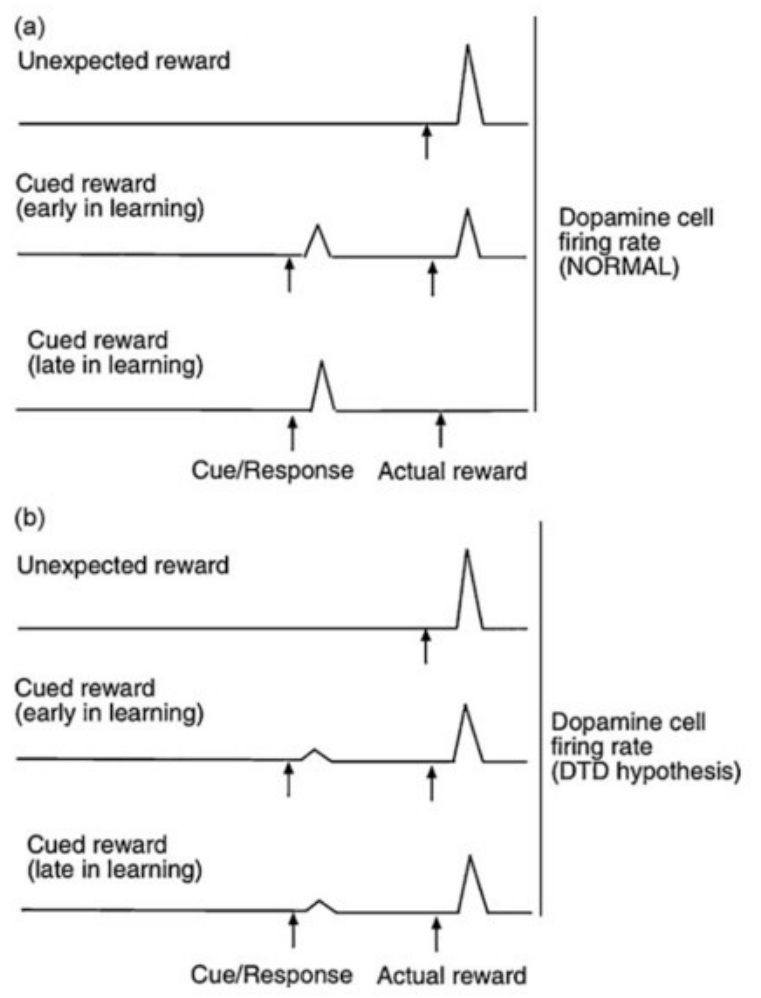

Figure 1. Typically, unexpected reinforcement and cued reward in early learning causes dopamine cells to fire, and there is still firing after the actual reward is received. Later in learning, responses to cues overpower responses to the reinforces. According to the dopamine transfer deficit theory, those with ADHD have dopamine cells that fail to fire in response to the cues that predict ensuing rewards. There is a sustained, substantial firing rate after the actual reward each time, proposing that the anticipatory midbrain dopamine system cell firing is disturbed. In patients with ADHD, the magnitude of the firing rate spikes remain generally sustained after each actual reward while spikes in normal patients subside after learning. The little arrows correspond to cue/response and actual reward. In normal controls, the spike of dopamine cell firing rate diminishes after the actual as the cued reward is learned over time, and increased firing rates are triggered after the cue/response instead. In contrast with those with ADHD, the dopamine cell firing 
rate stays relatively the same after the actual reward occurs, and the cue/response produces a significantly lower dopamine cell firing rate than normal even when the cue is supposed to be learned over time. So, there is more dopamine cell firing at the wrong time in ADHD patients. Reprinted from Tripp \& Wickens, 2008.

\section{Autism Spectrum Disorder}

Autism is a mental developmental disorder that affects social, imaginative, and communicative (speech and nonverbal) development. The syndrome is also commonly associated with attentional impairment, rapid and exaggerated changes in mood, impulsive/ repetitive behavior, and aggression. In the last three decades, autism has been investigated extensively and has become the most well-validated and extensively studied childhood mental disorders (Cook, 1990). The rather consistent natural developmental history of autism has been a significant aspect of neurochemical research. Gaze aversion, for instance, typically peaks anywhere between three to six years of age for autistic children, yet challenges involving social skills, language use, and abstract emotional concepts carry through both childhood and adulthood. Furthermore, over $30 \%$ of autistic children regress in development following initially normal development. Peripheral fluids and tissues have been the most investigated area of study, and the most consistent conclusion is that over $25 \%$ of autistic children are hyperserotonemic (having increased serotonin in the brain) (Cook, 1990). The effect of dopamine on autism, however, is also being increasingly investigated.

It is hypothesized that autistic behavior arises from abnormalities in the midbrain dopaminergic system. Mesocorticolimbic (MCL) circuit dysfunction leads to social deficits while nigrostriatal (NS) circuit dysfunction is responsible for stereotyped behaviors (both the MCL and NS are dopamine projection circuits) (figure 2) (Pavăl, 2017). The mesocorticolimbic circuit is often involved in reward and motivation, and dysfunction in this system can lead to decreased motivation to pursue rewards: in a social context, autistic patients may fail to comprehend certain social experiences as rewarding, leading to decreased motivation to pursue social interactions and develop socializing abilities (C. Chevallier et al., 2012). Consequently, the social motivation theory of autism spectrum disorder (involving MCL dysfunction) states that extreme cases of reduced social motivation affect social cognition, leading to these social deficits. In addition, the nigrostriatal system pathway is responsible for goal-oriented motor behavior, and a dysfunction in this circuit may lead to the entrapment into cycles of stereotyped, aimless patterns of behavior (C. Chevallier et al., 2012). Drug-induced NS circuit dysfunction caused stereotypies in mice studies: when mice were given intrastriatal D1 dopamine receptor antagonists, spontaneous stereotypies were attenuated, leading to the assumption that dopamine signaling abnormalities are involved in stereotypic behavior (Presti et al., 2003).

Additionally, a polymorphism of the dopamine 3 receptor gene, the dopamine 4 receptor gene, and the dopamine transporter in patients with autism are associated with increased striatal volume, resulting in increased repetitive motor patterns. These studies imply that a dopaminergic dysfunction within the NS circuit evokes stereotypic behavior.

The impact of this two-pronged dopamine hypothesis of Autism Spectrum Disorder is the eventual treatment using dopamine antagonists. Two such drugs, aripiprazole and risperidone, as of now, are the only Food and Drug Administration approved agents for autism (LeClerc \& Easley, 2015). They are used to resolve irritability, and several studies examined their effects on the core traits of Autism. Risperidone had a significant effect on stereotyped behavior and improved social behavior, measured by the Childhood Autism Rating Scale (Ghaeli et al., 2014). So, inhibiting dopamine transmission may lead to a behavioral improvement in general. These conclusions are limited, however, due to the massive heterogeneity of the disorder, affecting the results of clinical trials.

Furthermore, evidence for the dopamine hypothesis of autism is relatively scarce, so more research on humans specifically is essential for further understanding and impacts. 


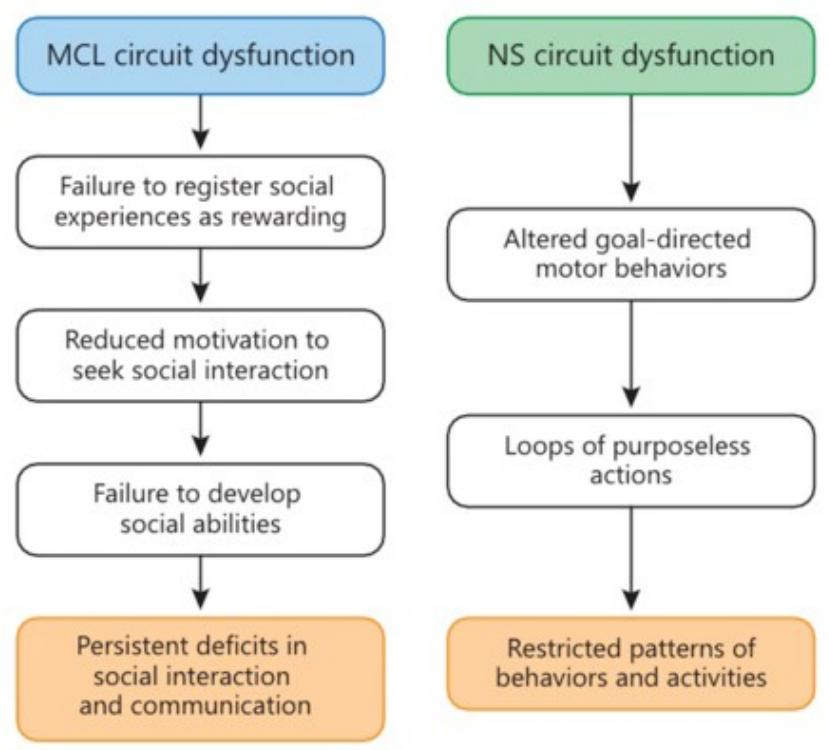

Figure 2. The study produces a two-pronged dopamine hypothesis framework as shown above. In the mesocorticolimbic (MCL) circuit, there is an alteration of dopamine signaling, specifically related to social reward. In the nigrostriatal system pathway (NS) circuit, abnormalities in dopamine receptor and transporter genes can lead to stereotyped autistic motor behaviors. The model explains which symptoms result from the two dopaminergic modulatory systems' dysfunctions. Reprinted from Pavǎl, 2017.

\section{Bipolar Disorder}

Bipolar Disorder (BD) is associated with manic episodes which may include symptoms such as having high levels of energy, a lesser need for sleep, and a disconnect from reality. Depressive episodes, however, are usually also symptomatic in BD patients and involve having low amounts of energy and motivation and decreased interest in daily activities. These episodes can last anywhere from days to months. Significant cognitive impairment can be closely associated with BD also as some people with BD struggle with verbal memory and tasks involving executive functions such as planning, set-shifting, and abstract thinking (Quraishi \& Frangou, 2002). There are two types of BD: I and II. BD I involves more severe mood episodes whereas BD II includes more moderate fluctuations in temperament. Due to BD becoming less stigmatized and more widely known, the dopamine theory of BD is becoming increasingly investigated.

Patients received two PET scans; the second scan involved oral administration of haloperidol lactate, which ultimately allowed for the quantifiable estimate of the D2 dopamine receptor density (D2 receptors regulate levels of dopamine in the synaptic cleft) (Pearlson et al., 1995). The study concluded that the density values of D2 receptors were higher for psychotic patients with bipolar disorder as compared to nonpsychotic patients with bipolar disorder and normal controls (psychosis is defined by the inability to distinguish between reality and imaginative thoughts, including having hallucinations or delusions) (figure 3). Although commonly associated with schizophrenia, the symptom can manifest in patients with bipolar disorder as well and can occur in both manic or depressive episodes. Patients' D2 receptor densities varied depending on the severity of the psychotic symptoms, yet the severity of nonpsychotic mood symptoms did not correlate with receptor density. Overall, it was concluded that psychotic patients with bipolar disorder showed elevations in D2 dopamine receptor density values, which are more correlated with psychosis than mood irregularity. Increased dopamine receptor densities may, thus, be an indication of psychotic symptoms.

Another study investigates striatal dopamine transporter availability in unmedicated patients with bipolar disorder. The dopamine transporter (DAT) is responsible for the regulation of dopamine neurotransmission and the reuptake of dopamine, which often dictates presynaptic dopamine function efficiency. DAT acts as an important 
marker for presynaptic function because it serves as the primary method of termination of dopamine transmission (which reduces the efficacy of dopamine transmission while undisrupted transmission occurs). The study uses PET scanning with the DAT-selective radiotracer [11C]CFT and MRI scans to research the difference of DAT availability between 11 bipolar disorder patients in either the manic or depressed phase and 13 healthy subjects. The unmedicated, diagnosed patients had much lower DAT availability as compared to the controls in the bilateral dorsal caudate, indicating abnormalities involving the dopaminergic system (Anand et al., 2011). These mesostriatal and mesolimbic dysfunctions may lead to issues with executive functioning, mood regulation, behavioral output, etc.

MRI studies also implied striatal activation and connectivity discrepancies in people with the disorder (Blumberg et al., 2005). In conclusion, dopamine receptor density and DAT proves to be an important area of increased investigation, especially to patients with bipolar psychosis.

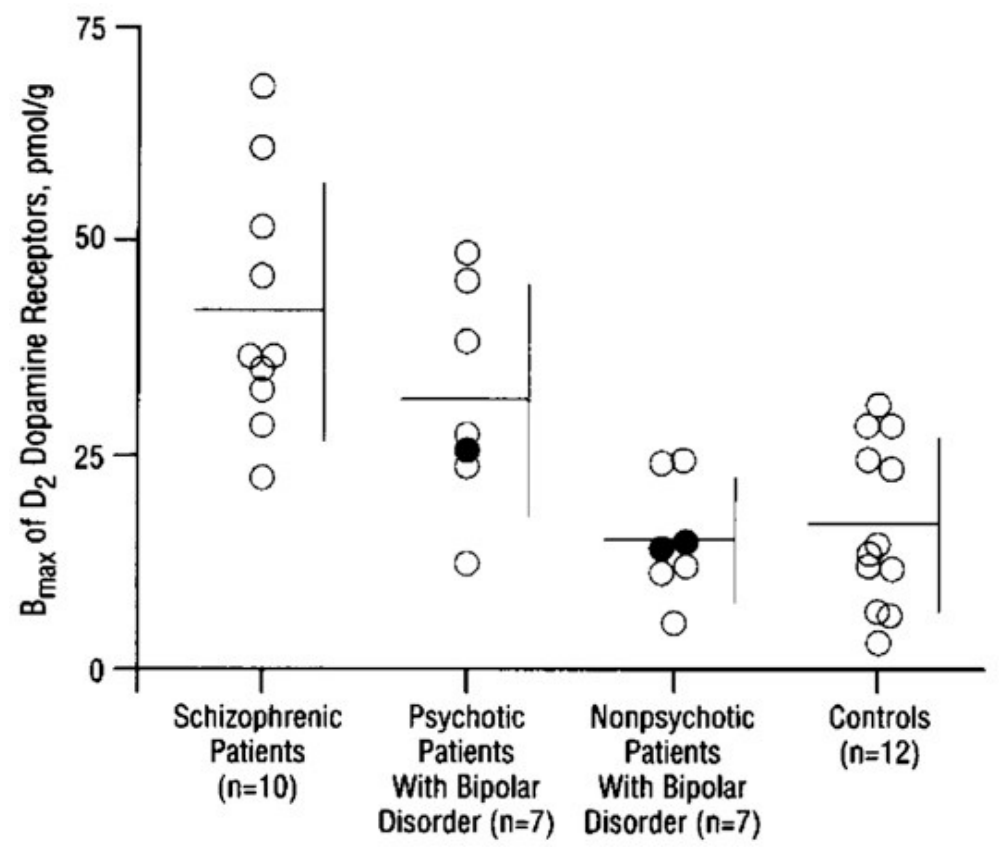

Figure 3. This scatterplot reflects the caudate and putamen D2 dopamine receptor density (Bmax) values for those with schizophrenia, psychotic patients with bipolar disorder, nonpsychotic patients with bipolar disorder, and normal controls found via PET scans. For the patients with bipolar disorder, open circles indicate manic patients while solid circles indicate depressed patients. The density of D2 dopamine receptors in psychotic patients with bipolar disorder is comparable with the density in schizophrenic patients. Reprinted from Pearlson et al., 1995.

\section{Major Depression}

Depression is a neuropsychiatric illness involving a constant feeling of sadness and a decreased level of excitement and energy for daily activities. Other symptoms may include changes in sleep patterns, loss of appetite and self-esteem, difficulty concentrating, and functional impairment. This illness is also associated with a greater risk of mortality and suicide. Depression is the most common mood disorder with a lifetime prevalence estimated at around $7 \%$ to $21 \%$ (Vilagut et al., 2016). Major depression, therefore, is among the most burdensome of health issues at the individual and societal scale. Around 50\%, however, of patients with depression are hypothesized to be falsely identified by general practitioners, who are unassisted with depression diagnosis (Mitchell et al., 2009). 
Additionally, only a limited amount of those with depression receive proper and effective treatment. So, depression remains and continues to be an area of much medical debate and investigation.

The dysfunction of the dopamine system in depression plays a central role in the inability to feel enjoymentanhedonia. This symptom also incorporates the complicated reward-related deficits patients with depression possess, including abnormalities in motivation, anticipation, and decision-making processes involving reward, which the dopamine system has a direct correspondence to. In depressed patients with anhedonia, PET imaging studies reflected significantly lower dopamine transporter binding as compared to controlled subjects, suggesting downregulation of dopamine concentration (Belujon \& Grace, 2017). Studies also depict a decreased dopamine transporter density when dopamine itself is depleted, worsening the issue. Earlier studies of postmortem depressed patients show an increased striatal D2 receptor binding in the central and basal nuclei of the amygdala, suggesting abnormal dopaminergic activity (Belujon \& Grace, 2017).

Dopaminergic activity is involved in multiple regions of the brain such as the ventral subiculum, basolateral amygdala, and hippocampus. The dysfunction in the dopamine system in relation to depression likely stems from the dopaminergic system's regulatory afferent circuits, which consist of afferent neurons referring to nerve cells that carry information into the central nervous system. Spontaneously active dopamine neurons can fire in an irregular, slow pattern (tonic activity) or in rapid bursts of action potentials (phasic activity) (Floresco et al., 2003). Tonic dopamine activity is associated with two opposing circuits (figure 4): an activating circuit, which involves the nucleus accumbens and the hippocampus, as well as an inhibitory circuit involving the basolateral amygdala. When responding to important stimuli, large proportions of dopamine neurons will be activated, causing a strong burst in dopamine release, and the amygdala decreases the responsiveness of the reward-related dopamine system. Abnormalities within this dopamine pathway lead to problems in those with major depression.

\section{Control}

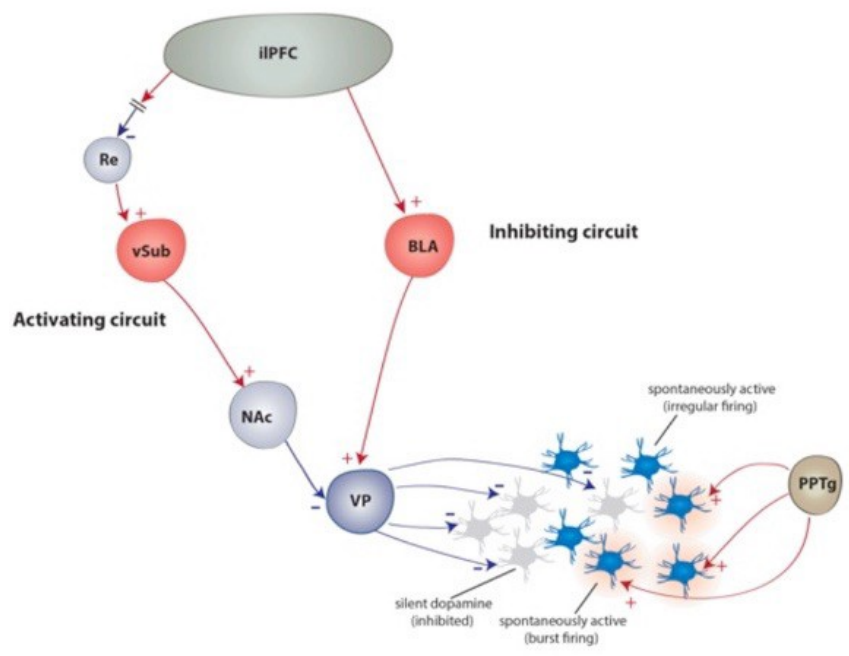

Figure 4. In normal control patients, the dopaminergic system is regulated by an inhibitory circuit including the basal lateral amygdala (BLA)-ventral pallidum (VP) pathway which is activated by the infralimbic sub- region(ilPFC), the Re-ventral subiculum of the hippocampus (vSub)- nucleus accumbens (NAc)- VP pathway that is inhibited by the ilPFC. Only dopaminergic neurons that fire spontaneously can fire in response to rapid activation with bursts of action potentials, due to the pedunculopontine tegmental nucleus (PPTg) afferents. Modified from Belujon \& Grace, 2017.

The chronic mild stress (CMS) model is one of the most extensively validated models of major depression, and CMS in rodents have shown a prolonged decrease of dopamine in the ventral tegmental area (Dziedzicka- 
Wasylewska M; Willner, P; Papp, 1997). Inactivation of the infralimbic subregion suggests hyperactivity of pathways responsible for downregulating the dopamine system in depression, measured by a decrease in the number of dopamine neurons firing spontaneously (figure 5). Also, too much inhibitory activity weakens the excitation of dopamine activating structures like the nucleus accumbens. This conclusion is upheld by the learned helplessness model, another widely accepted model of the disorder (Kram et al., 2002). Here, the rats get exposed to inescapable stressors such as shocks. Yet, when reconditioned to these stressors, even with a means to easily escape, the animals lose the ability to demonstrate escape behavior: a learned helplessness. Both of these models (CMS and learned- helplessness) contribute to observing major depression using models proposing altered striatal dopaminergic activity. 


\section{Depression}

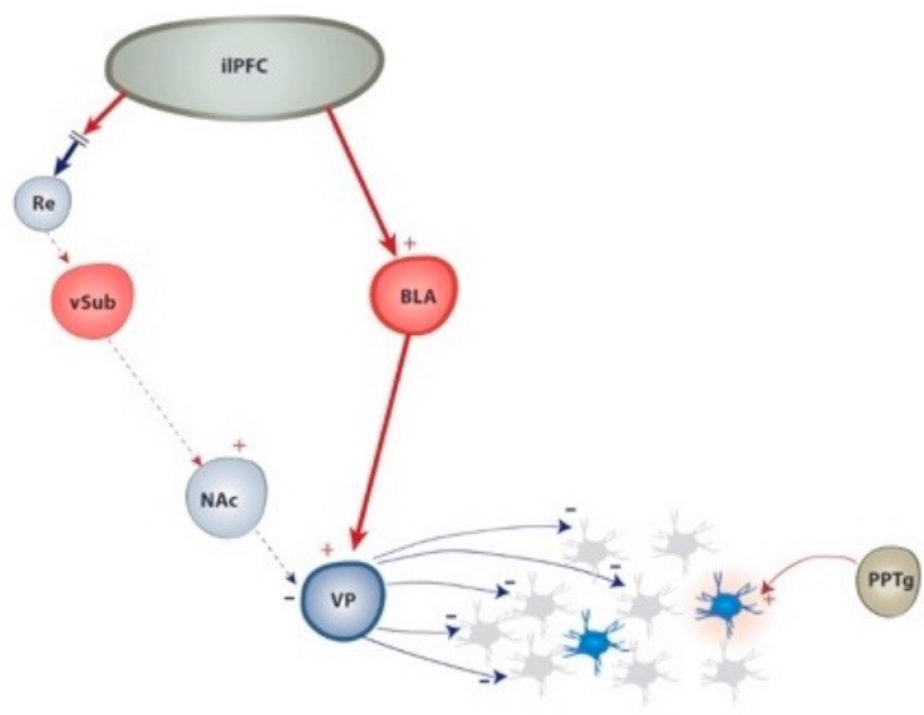

Figure 5. In depressive patients, the dopamine system is downregulated as measured by a decrease in the number of dopamine neurons that fire spontaneously. The decrease is due to the hyperactivity of the infralimbic subregion, activating inhibition in the basolateral amygdala (BLA)-ventral pallidum (VP) pathway and increasing hyperactivity through the Re-ventral subiculum of the hippocampus (vSub)-nucleus accumbens (NAc)-VP pathway. Thus, there are fewer bursts of action potentials due to less activation of pedunculopontine tegmental nucleus (PPTg) afferents. Modified from Belujon \& Grace, 2017.

\section{Post Traumatic Stress Disorder}

Post Traumatic Stress Disorder (PTSD) is characterized by an exposure to a traumatizing event leading to a cluster of negative symptoms such as flashbacks and nightmares of the event, hyperactivity (like exaggerated responses to threatening stimuli), and tendencies to distance oneself from situations similar to the scarring experience itself. PTSD patients' hyperarousal is demonstrated by increased heart rate, respiratory rate, galvanic skin response, and an overall lack of ability to inhibit exaggerated emotional responses (Krystal et al., 1989).

Although the scientific investigation of PTSD originated in the 1910s following the horrors of World War I when many veterans suffered from PTSD, research eventually halted because scientists failed to identify the brain lesions of traumatized patients. Not until the 1960s did psychophysiological PTSD studies begin again, with treatments lagging significantly behind other mental disorders like schizophrenia and depression. Today, an increased investigation is devoted to locating derangements within the threat-caused circuitry, and PTSD studies are on the rise.

Hyperactive fear circuits, like the amygdala system, are the basis for PTSD comparable to a "stuck accelerator." Dopamine, traditionally known for motivation, addiction, and reward prediction, is also increasingly related to controlling fear and anxiety. There are multiple subtypes of dopaminergic neurons with different temporal dynamics, which makes the exact role of dopamine in anxiety-resistance unknown. However, the rebound-excitation theory explains that dopaminergic neurons' rebound then excitation at the end of a scarring experience is the "brake" to the "stuck accelerator" in PTSD patients (Lee et al., 2016). In normal controls, dopaminergic neurons can rebound after a jarring experience and, thus, provide an intrinsic safety signal to fear-processing neural circuits in a spatially and temporally controlled manner. In patients with PTSD, this rebound or this "brake" fails to occur properly, resulting in a "stuck accelerator" of hyperactive fear circuits. Dopamine may provide important safety signals to neural circuits that trigger fear. These rebound-excitation dopaminergic neurons may be influenced by experiences and genetics, which can serve as a precursor to predicting individual resilience to stress (Yehuda et al., 2006). 
To observe the way dopaminergic neurons respond to traumatic events, replications of earthquakes and free falls were made on mice (Liu et al., 2014). Using pharmacological and optogenetic methods, in vivo recordings of dopaminergic activity showed both fear-inhibited and fear-excited dopaminergic neurons. Aversive-inhibited dopamine neurons demonstrated rebound-excitation as a result of unexpected aversive stimuli, which leads to the belief that sub-population of neurons function as safety signals in response to apprehensive events (figure 6) (Wang \& Tsien, 2011). The signaling strength of phasic dopamine's release affects short and long-term termination for fearful stimuli. The phasic dopamine was "time-locked," in the shocked mice, resulting in long-term changes in response to fear (Lee et al., 2016). So, abnormality in the dopamine rebound-excitation may present itself as PTSD symptoms-for example, flashbacks-because the lack of rebound safety signal leads to stress susceptibility and reduced clearance of fear memory traces.

Dopamine neurons are also known to project into the prefrontal cortex, a crucial emotional information processing center. Neurons in the anterior cingulate cortex showed a wide range of responses in response to traumatizing events mimicking experiences in combat. This pattern reverberation, a process in which real-time memory patterns get replayed quickly after emotionally-charged events, often affects blast-exposed animals (Xie et al., 2013). Dopamine rebound-excitation may change pattern reverberation of these anxiety-provoking memories in the anterior cingulate cortex and hippocampus. A limitation to the rebound-excitation theory is that present imaging techniques such as fMRI still have limited spatial and temporal resolution, which can make rebound-excitation research in human beings challenging (Lee et al., 2016). Regardless, abnormality in dopamine rebound-excitation seems relatively relevant to the hyperactivity of fear circuitry in those suffering from PTSD.

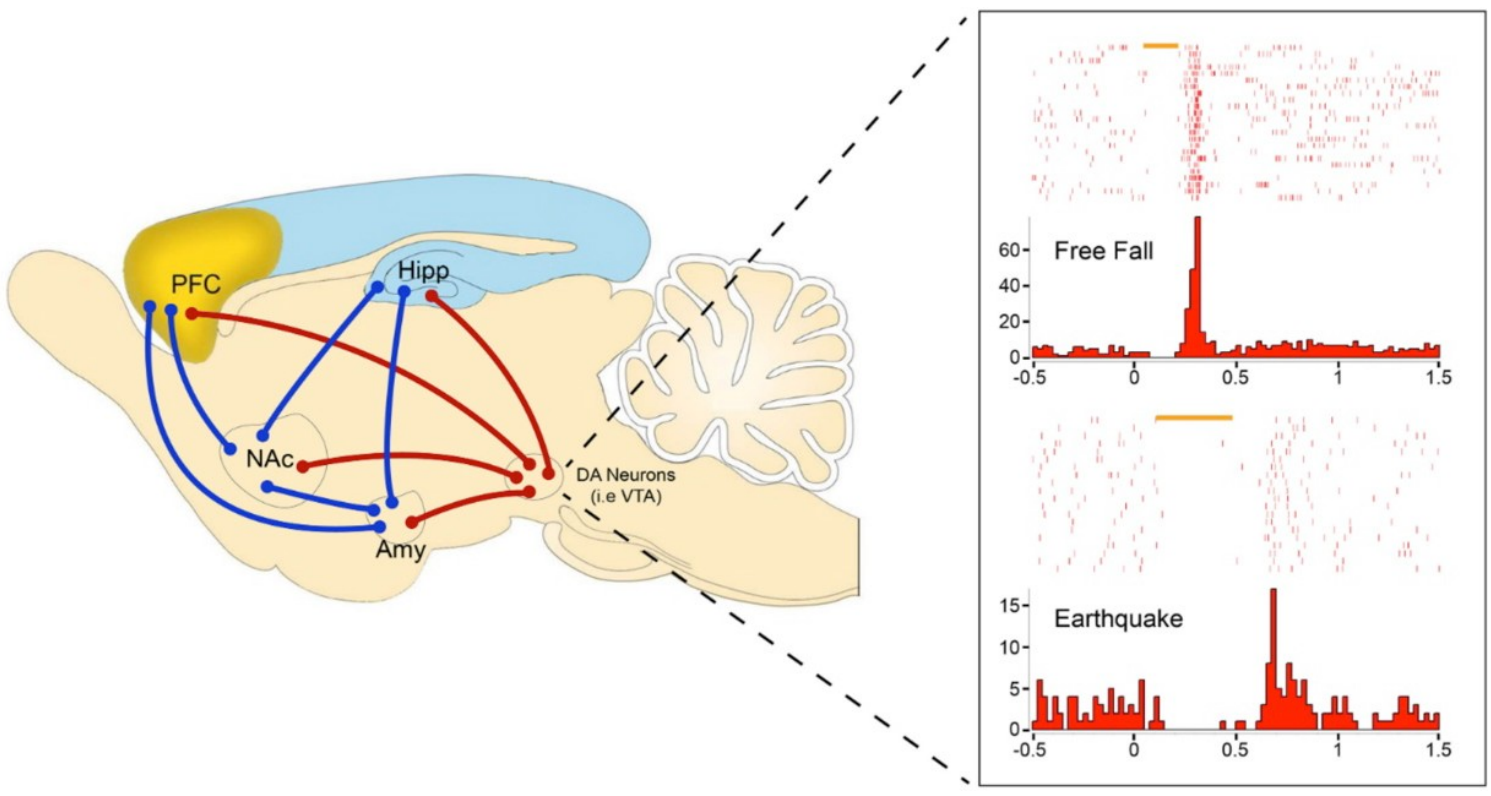

Figure 6. To specifically examine how Dopaminergic neurons react to traumatizing real-life events, the laboratory used fearful unconditional stimuli such as free falling and earthquakes to test on mice. The amygdala fear circuit is blue whereas the Dopaminergic circuit is red. Many aversive-inhibited dopaminergic had phasic rebound-excitation reactions to unexpected stimuli. This pattern led to the idea that offset phasic rebound excitation of dopaminergic neurons may occur as a safety signal, broadcasting down to target areas such as the amygdala (Amy), nucleus accumbens (NAc), prefrontal cortex (PFC), or hippocampus (Hipp). Reprinted from Lee et al., 2016. 


\section{Schizophrenia}

Schizophrenia is a mental illness consisting of three primary symptoms: positive, negative, and cognitive.

Positive symptoms, which are symptoms with exaggerated effects, include delusions, disorganized speech or behavior, and hallucinations. Negative symptoms, also known as the "flat affect," are giving inappropriate responses, alogia, and avolition. Furthermore, cognitive symptoms include difficulty with memory, learning, and understanding. Despite getting optimal treatment, two-thirds of schizophrenic patients still suffer persisting or fluctuating symptoms (Saha et al., 2005). Studies report that men, migrants, and those living in urban environments are more susceptible to Schizophrenia as compared to women, native-born individuals, and those residing in rural settings. Though the prevalence of this disorder is relatively low, affecting about .4\% of the population, Schizophrenia still remains a major global issue (Saha et al., 2005). The dopamine hypothesis of schizophrenia (or the dopamine hypothesis of psychosis) is a model proposing that the symptoms triggered by this mental illness are closely related to a hyperactivation of the dopaminergic signal transduction system.

The projection of dopaminergic neurons extending from the core of the ventral tegmental area as well as the substantia nigra to the striatal complex has a substantial effect on those with schizophrenia. The striatal complex allows for inhibition of the thalamus, which filters sensory information, but dopamine D2 receptors in the complex inhibit output going to the thalamus (Carlsson, 2006). This abnormal inhibition triggers hyperactivity of the dopamine system, which in turn, inhibits the inhibitory influence of the receptors at the thalamus. So, the thalamus experiences hyperactivity, which over-stimulates the cortex. Additionally, dopaminergic synapses in schizophrenic patients are essentially defective. The less effective synaptic transmission then triggers dopaminergic neurons through feedback loops so that intact dopaminergic extrasynaptic transmission trumps transmission (figure 7). This extrasynaptic dopaminergic transmission occurs as a mechanism to compensate for the poor synaptic transmission itself. Positive symptoms may thus occur due to increased extrasynaptic, and thus, more poorly controlled transmission while negative symptoms and abnormal cognitive behavior result because of worse compensation of synaptic defects. In regards to antipsychotic drugs used for treatment, antipsychotics usually inhibit both synaptic and extrasynaptic transmission, which do result in less psychotic action but also the intensifying of negative symptoms and cognitive defects. Dopaminergic stabilizers specifically are designed to inhibit only extrasynaptic transmission but uphold synaptic transmission, facilitating in the decrease of both positive and negative symptoms and improving cognition (Carlsson, 2006). These stabilizers have been proved to be helpful in restoring social behavior in a rat model of schizophrenia but have yet to be thoroughly examined in humans (Rung et al., 2005).

A study consisting of a compilation of 193 peer-reviewed MRI studies ranging from the year 1988 to 2000 allows for more definitive endings of brain abnormalities in schizophrenia patients (Shenton et al., 2001). Advances in in vivo MRI techniques have shown abnormalities in the frontal, parietal, and occipital lobes as well as the cerebellum and subcortical structures. $68 \%$ of studies reviewed basal ganglia dysfunctions, which correlates most strongly with dopamine function. Structures within the basal ganglia, such as the caudate, putamen, and globus pallidus, are relevant to schizophrenia research due to the extensive dopaminergic inputs to them. Although the first postmortem study of these structures proved no abnormalities in schizophrenics, another study later found that the bilateral volume in the striatum increased by $9 \%$ and the volume of the globus pallidus increased by $14 \%$ in the globus pallidus (Heckers et al., 1991). Additionally, there have been 25 MRI studies of the basal ganglia and its relevance to schizophrenia. 17 MRI studies confirm positive findings whereas eight report negative findings. Most positive findings reported increases in volume. One study confirmed a 5.7\% increase in caudate size is likely due to neuroleptic exposure whereas atypical neuroleptics, which diminished effects on D2 receptors, caused a decrease in caudate size (Chakos et al., 1994). This evidence suggests that increased volume in basal ganglia structures are due to neuroleptics (also known as antipsychotics which depress nerve functions and ease symptoms of hallucinations and delusions) and not atypical neuroleptics.

Another study quantified dopamine D2 receptor densities and superior temporal gyrus using both MRI and PET scans as both the temporal lobe and basal ganglia have proved relevant to schizophrenia (Tune et al., 1996). 
The MRI's purpose was to measure the volume of the superior temporal gyrus while the PET scans served to estimate striatal dopamine D2 receptor density. Fourteen chronic schizophrenic patients and fifteen controlled patients were involved in the experiment. The MRI found that the average left and right superior temporal gyral volumes were found to be smaller in the schizophrenic group, and PET scans found that average striatal densities were higher in the schizophrenic patients. Together, the study proves an inverse relationship between decreased superior temporal gyral volume and increased striatal D2 receptor density values. The study also revealed reduced left anterior temporal lobe metabolism as well as abnormal cerebral blood flow in the parahippocampal gyrus, which are associated with positive symptoms (Buchsbaum, 1990). Additionally, basal ganglia abnormalities are increased blood flow in left globus pallidus and decreased basal ganglia metabolism. A limitation of this study is that only a total of 29 people were involved- larger groups of patients and control subjects are needed to confirm these findings. Overall, although there are different methods and approaches to explaining the dopamine hypothesis of schizophrenia, more investigation into dopamine transmission, receptors, and structures are crucial in developing more effective medication. 


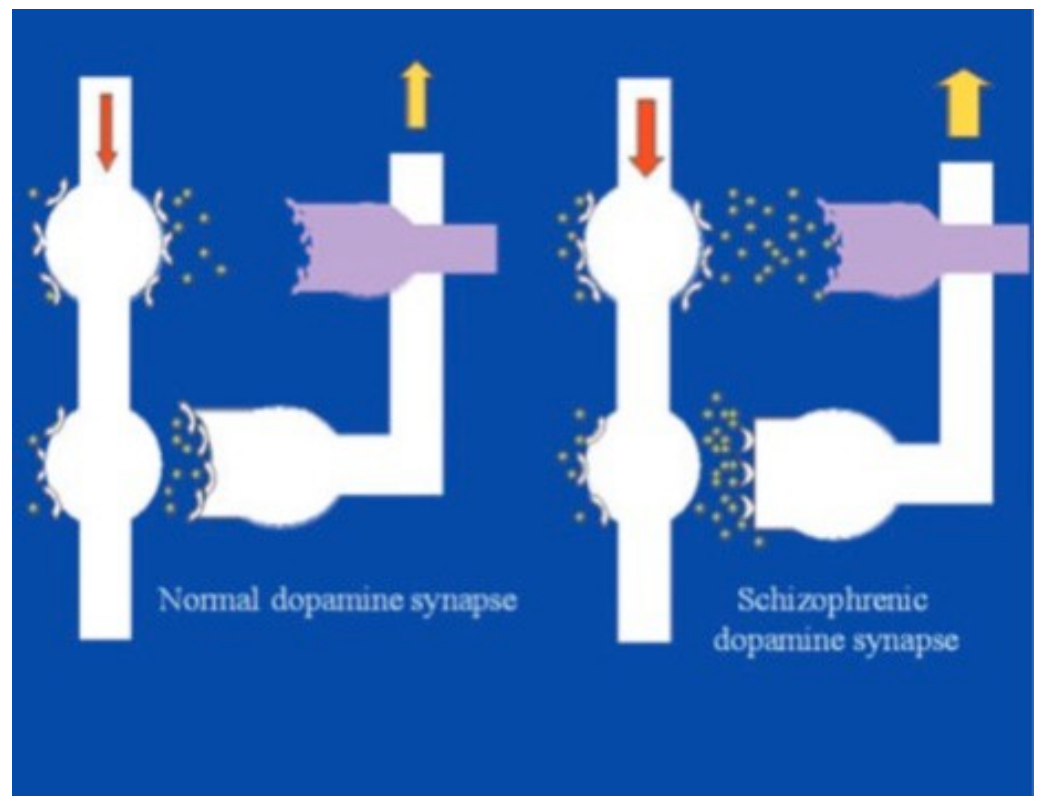

Figure 7. It is proposed that, in schizophrenia, dopaminergic synapses are defective, and inefficient synaptic transmission then leads to more activated dopaminergic neurons through feedback loops as compensation. So, extrasynaptic transmission occurs over a portion of transmission. Therefore, positive symptoms may result because of extrasynaptic and less controllable dopamine transmission. Cognitive deficits and negative symptoms, in contrast, may be because of poor compensation of synaptic defects. Reprinted from Carlsson \& Carlsson, 2006.

\section{Conclusion}

The dopamine system and its dysfunction are correlated with many different psychiatric disorders such as ADHD, Autism, Bipolar Disorder, Depression, PTSD, and Schizophrenia. The dopamine hypothesis of schizophrenia remains the most widely accepted as neuroleptic drugs relieving psychosis have been studied to block D2 dopamine receptors in the caudate nucleus, suggesting an abnormal increase in receptors. Many other disorders and their relation to the neurotransmitter, however, are also beginning to be increasingly studied as well because of the similarity of the clinical symptoms of schizophrenia and other mental illnesses.

Positive symptoms like hallucinations, delusions, and thought disorder in schizophrenia are also prevalent in patients with bipolar psychosis. Psychotic patients with bipolar disorder exhibited higher dopamine D2 receptors similar to aged patients with schizophrenia (Pearlson et al., 1995). This increase in receptor density can lead to increased transmission of dopamine, resulting in psychotic symptoms. Another study investigates the dysfunction of the striatal dopamine transporter (DAT) in patients with BD, and through PET scans, discovered that there was lower DAT availability (Anand et al., 2011). DAT is also important to the termination of dopamine transmission, so having less of the transporter can lead to increased dopamine levels, resulting in problems with executive function and mood regulation. Negative symptoms in schizophrenics such as alogia and avolition are also common symptoms of depressive episodes in those with bipolar disorder and depression. A study of major depression observed that there was lower dopamine transporter binding and density in depressive patients (also seen in patients with BD), which causes a downregulation of dopamine (Saha et al., 2005). The chronic mild stress model showed a longer decrease of dopamine in the ventral tegmental area because of a decrease in dopamine neurons that fire spontaneously, resulting in alogia and avolition Dziedzicka-Wasylewska M; Willner, P; Papp, 1997).

Autism and schizophrenia, which share social behavioral traits such as social withdrawal and lack of emotional response, are both thought to possess subcortical dopamine excess as well as cortical dopamine deficit. The shared 
behavior may indicate a similar pathogenic mechanism, but there is still a lack of evidence to confirm the dopaminergic model of autism: the mesocorticolimbic circuit dysfunction affecting social reward and the nigrostriatal system circuit dysfunction resulting in altered goal-directed motor behaviors (Pavǎl, 2017).

Schizophrenic symptoms of hyperactivity can intersect with symptoms experienced by people with ADHD and PTSD as well. The two theories of ADHD are the dynamic developmental theory (DDT) and the dopamine transfer deficit theory (DTD). The DDT hypothesizes abnormality involving dopamine transmission in the frontolimbic brain circuitry, ending in shorter dopamine firing time and lesser feeling of reward when appropriate (Luman et al., 2010). The basis of the dysfunction in transmission is due to lower tonic dopamine amounts. In contrast, the DTD proposes that tonic levels are normal but abnormal timing of dopamine cell firing is the cause of inaccurate prediction of reward in those with ADHD. Either way, both theories result in people with ADHD prioritizing weaker, more immediate rewards over long-term rewards. This behavior leads to the symptoms of impulsivity, poor planning, and disorganization.

Dopamine's role in PTSD has more correlation with dopamine's role in providing safety signals to pacify neural circuits that prompt fear. Phasic dopamine in response to a fearful event may be "time-locked," resulting in an inability to suppress the feeling of apprehension (Lee et al., 2016). This lack of rebound-excitation in dopamine neurons leads to hyperactivity of fear circuits and, thus, symptoms such as flashbacks.

Further investigation in the area of the dopamine system will prove beneficial in discovering better treatment for a wide range of psychiatric disorders. There are different medications intended to normalize various aspects of the dopamine system. Many antipsychotic drugs act upon dopamine D2 receptors found in the striatal complex to lessen psychotic symptoms, especially used in schizophrenia. Typical antipsychotics inhibit extrasynaptic and synaptic transmission, which leads to worsening cognitive abnormalities and negative symptoms (Carlsson, 2006). Dopaminergic stabilizers such as the substituted phenylpiperidine (-)-OSU6162 and a related compound, ACR16, bind to the receptors but demonstrate a relatively low affinity for dopamine D2 receptors in vitro, which allows them to inhibit extrasynaptic transmission and encourage synaptic transmission (Carlsson \& Carlsson, 2006). This way, there is an improvement in both antipsychotic action and general cognition and negative symptoms. Other atypical antipsychotics like clozapine and aripiprazole and partial dopaminergic antagonists are an area of further research (Carlsson \& Carlsson, 2006).

Dopamine, as of now, is a matter of correlation and not causation in many of these disorders with the exception of schizophrenia. Since many of the studies reviewed were either the very first dopamine hypothesis of that specific disorder or one of very few original studies investigating dopamine's involvement in the disorder- exclusively-it is unclear whether the neurotransmitter's involvement is the sole cause of the various positive, negative, cognitive, and social symptoms manifesting in people with these psychiatric illnesses. Another limitation is that, in most of the studies' methods, there were only one or two dozen test subjects, and the results have yet to be confirmed in larger cohorts. Furthermore, there is still an ongoing debate about quantitative PET measurements of dopamine receptors. In Farde's study investigating schizophrenia, a different radioligand (the antagonist C- Raclopride) that is a very selective D2 dopamine receptor antagonist and an in vivo saturation procedure was used to measure the density of receptors (Farde et al., 1987). The study could not confirm an increase in D2 dopamine receptors like most other studies using PET to measure D2 dopamine receptor density, such as Wong's study (which used haloperidol-induced inhibition of UC-N-Methylspiperone binding) and propose a further investigation into different PET methods (Tune et al., 1996). All of these studies did, indeed, demonstrate some sort of abnormality with either dopamine firing rate, density, and extrasynaptic dopamine transmission in relation to the disorder.

Therefore, dopamine does, indeed, affect the six prevalent psychiatric illnesses of ADHD, autism, bipolar disorder (with psychosis), major depression, PTSD, and schizophrenia. 


\section{Acknowledgments}

I would like to thank the Horizon Academic Research Program for introducing me to my mentor Julian Day-Cooney and granting me with this research opportunity. This project could not have been accomplished without their patience and support. Additionally, I would like to express gratitude for my family and friends who helped me persist through the learning and researching process.

\section{Reference Table}

\begin{tabular}{|c|c|c|c|}
\hline $\begin{array}{c}\text { Name of } \\
\text { the Tech- } \\
\text { nique }\end{array}$ & Description & Benefits & Limitations \\
\hline $\begin{array}{c}\text { functional } \\
\text { magnetic } \\
\text { resonance } \\
\text { imaging (fMRI) }\end{array}$ & $\begin{array}{l}\text { fMRI is a medical } \\
\text { imaging technique used in } \\
\text { radiology to create } \\
\text { pictures of anatomy and } \\
\text { physiological processes. } \\
\text { fMRI measures brain } \\
\text { activity by detecting } \\
\text { changes in blood flow } \\
\text { and } \\
\text { is used to observe the } \\
\text { brain's functional } \\
\text { anatomy. }\end{array}$ & $\begin{array}{c}\text { - high spatial resolution } \\
\text { - Non-invasive } \\
\text { - Practical and easy to } \\
\text { use } \\
\text { - can be repeatedly used } \\
\text { by the same individual } \\
\text { - can image the whole } \\
\text { brain }\end{array}$ & $\begin{array}{c}\text { - expensive: on average, a } \\
\text { procedure costs } \$ 400-\$ 3500, \text { and } \\
\text { an average machine costs over } \\
\$ 1,000,000 \\
\text { - may be inaccurate: slight } \\
\text { movement affects quality of } \\
\text { image } \\
\text { - measures blood flow only and } \\
\text { not the aggregate activity of } \\
\text { millions of neurons } \\
\text { - poor temporal resolution }\end{array}$ \\
\hline $\begin{array}{c}\text { positron emission } \\
\text { tomography } \\
(\text { PET })\end{array}$ & $\begin{array}{c}\text { a PET scan uses a } \\
\text { radioactive drug to } \\
\text { visualize and to measure } \\
\text { the metabolic processes in } \\
\text { the body. }\end{array}$ & $\begin{array}{l}\text { - can limit imaging brain } \\
\text { activity in more specific } \\
\text { areas than fMRI } \\
\text { - Able to detect early } \\
\text { stages of diseases before } \\
\text { symptoms show e.g. } \\
\text { epilepsy } \\
\text { - Studies metabolic } \\
\text { functions: alternative to } \\
\text { biopsy } \\
\text { - can image the whole } \\
\text { brain }\end{array}$ & $\begin{array}{c}\text { - ionizing radiation: relatively low } \\
\text { radiation but not recommended to } \\
\text { be used frequently } \\
\text { - expensive: one brain PET scan } \\
\text { can cost an average of } \$ 3,000- \\
\$ 4,000 \\
\text { - radioactive compound is short- } \\
\text { lived } \\
\text { - radioisotope must be produced } \\
\text { in } \\
\text { laboratory near PET scanner } \\
\text { - lower spatial resolution } \\
\text { - poor temporal resolution }\end{array}$ \\
\hline
\end{tabular}




\section{References}

Anand, A., Barkay, G., Dzemidzic, M., Albrecht, D., Karne, H., Zheng, Q. H., Hutchins, G. D., Normandin, M. D., \& Yoder, K. K. (2011). Striatal dopamine transporter availability in unmedicated bipolar disorder. Bipolar Disorders, 13(4), 406-413. https://doi.org/10.1111/j.1399-5618.2011.00936.x

Arias-Carrián, O., Stamelou, M., Murillo-Rodríguez, E., Menéndez-Gonzlez, M., \& Pöppel, E. (2010). Dopaminergicreward system: A short integrative review. International Archives of Medicine, 3(1), 1-6.

https://doi.org/10.1186/1755-7682-3-24

Belujon, P., \& Grace, A. A. (2017). Dopamine system dysregulation in major depressive disorders. In InternationalJournal of Neuropsychopharmacology (Vol. 20, Issue 12, pp. 1036-1046). Oxford University Press. https://doi.org/10.1093/ijnp/pyx056

Blumberg, H. P., Fredericks, C., Wang, F., Kalmar, J. H., Spencer, L., Papademetris, X., Pittman, B., Martin, A., Peterson, B. S., Fulbright, R. K., \& Krystal, J. H. (2005). Preliminary evidence for persistent abnormalities inamygdala volumes in adolescents and young adults with bipolar disorder. Bipolar Disorders, 7(6), 570-576. https://doi.org/10.1111/j.1399-5618.2005.00264.x

Buchsbaum, M. S. (1990). The Frontal Lobes, Basal Ganglia, and Temporal Lobes as sites for schizophrenia. Schizophrenia Bulletin, 16(3), 379-389. https://doi.org/10.1093/schbul/16.3.379

C. Chevallier, Kohls, G., Troiani, V., Brodkin, E. S., \& Schultz, R. T. (2012). The Social Motivation Theory. Trends inCognitive Sciences, 16(4), 231-239. https://doi.org/10.1016/j.tics.2012.02.007.The

Carlsson, A. (2006). The neurochemical circuitry of schizophrenia. Pharmacopsychiatry, 39(SUPPL. 1). https://doi.org/10.1055/s-2006-931483

Carlsson, A., \& Carlsson, M. L. (2006). A dopaminergic deficit hypothesis of schizophrenia: the path to discovery. In

Dialogues Clin Neurosci (Vol. 8). www.dialogues-cns.org

Chakos, M. H., Lieberman, J. A., Bilder, R. M., Borenstein, M., Lerner, G., Bogerts, B., Wu, H., Kinon, B., \& Ashtari,

M. (1994). Increase in caudate nuclei volumes of first-episode schizophrenic patients taking antipsychotic drugs.

American Journal of Psychiatry, 151(10), 1430-1436. https://doi.org/10.1176/ajp.151.10.1430

Cook, E. H. (1990). Autism: Review of Neurochemical lnvestigation. In SYNAPSE.

Dziedzicka-Wasylewska M; Willner, P; Papp, M. (1997). Changes_in_dopamine_receptor_mRNA_expression.17.pdf.Farde, L., Wiesel, F.-A., Halldin, C., \& Stone-Elander, S. (1987). No D2 Receptor Increase in PET Study of

Schizophrenia To the Editor. $\backslash p=m$-Densities of D2 dopamine receptors in postmortem.

https://jamanetwork.com/ 
Floresco, S. B., West, A. R., Ash, B., Moorel, H., \& Grace, A. A. (2003). Afferent modulation of dopamine neuron firingdifferentially regulates tonic and phasic dopamine transmission. Nature Neuroscience, 6(9), 968973. https://doi.org/10.1038/nn1103

Ghaeli, P., Nikvarz, N., Alaghband Rad, J., Alimadadi, A., \& Tehrani Doost, M. (2014). Effects of risperidone on coresymptoms of autistic disorder based on childhood autism rating scale: An open label study. Indian Journal of Psychological Medicine, 36(1), 66-70. https://doi.org/10.4103/0253-7176.127254

Heckers, S., Heinsen, H., Heinsen, Y., \& Beckmann, H. (1991). Cortex, white matter, and basal ganglia in schizophrenia:A volumetric postmortem study. Biological Psychiatry, 29(6), 556-566.

https://doi.org/10.1016/0006- 3223(91)90091-Y

Kram, M. L., Kramer, G. L., Ronan, P. J., Steciuk, M., \& Petty, F. (2002). Dopamine receptors and learned helplessnessin the rat. Progress in Neuro-Psychopharmacology and Biological Psychiatry, 26(4), 639-645. https://doi.org/10.1016/s0278-5846(01)00222-6

Krystal, J. H., Kosten, T. R., Mason, J. W., Perry, B. D., \& Giller, E. L. (1989). Neurobiological Aspects of I'ISD:Review of Clinical and Preclinical Studies STEVEN SOU i ltW1CK. In BEHAVIOR THERAPY (Vol. 20),

LeClerc, S., \& Easley, D. (2015). Pharmacological therapies for autism spectrum disorder: A review. $P$ and T, 40(6),389-397.

Lee, J. C., Wang, L. P., \& Tsien, J. Z. (2016). Dopamine rebound-excitation theory: Putting brakes on PTSD. Frontiers inPsychiatry, 7(SEP). https://doi.org/10.3389/fpsyt.2016.00163

Liu, J., Wei, W., Kuang, H., Tsien, J. Z., \& Zhao, F. (2014). Heart rate and heart rate variability assessment identifies individual differences in fear response magnitudes to earthquake, free fall, and air puff in mice. $P L o S$ ONE, 9(3).https://doi.org/10.1371/journal.pone.0093270

Luman, M., Tripp, G., \& Scheres, A. (2010). Identifying the neurobiology of altered reinforcement sensitivity in ADHD:A review and research agenda. Neuroscience and Biobehavioral Reviews, 34(5), 744-754.

https://doi.org/10.1016/j.neubiorev.2009.11.021

Mandal, A. (2019). News Medical. Retrieved October 9, 2020, from https://www.newsmedical.net/health/Dopamine-Functions.aspx

Mitchell, A. J., Vaze, A., \& Rao, S. (2009). Clinical diagnosis of depression in primary care: a meta-analysis. The Lancet,374(9690), 609-619. https://doi.org/10.1016/S0140-6736(09)60879-5

NIH. (2017). Parkinson's Disease.

Pavăl, D. (2017). A Dopamine Hypothesis of Autism Spectrum Disorder. In Developmental Neuroscience (Vol. 39, Issue5, pp. 355-360). S. Karger AG. https://doi.org/10.1159/000478725

Pearlson, G. D., Dean, B. ;, Wong, F., Tune, L. E., Ross, C. A., Chase, G. A., Links, J. M., Dannals, R. F., Wilson, A. A.,Ravert, H. T., Wagner, H. N., \& Depaulo, ; J Raymond. (1995). In Vivo D2 Dopamine Receptor 
Density in Psychotic and Nonpsychotic Patients With Bipolar Disorder Background: A prior positron emission tomographic study from The. https://jamanetwork.com/

Polanczyk, G., Silva de Lima, M., Lessa Horta, B., Biederman, J., \& Augusto Rohde, L. (2007). Article The WorldwidePrevalence of ADHD: A Systematic Review and Metaregression Analysis. In Am J Psychiatry (Vol. 164).

Presti, M. F., Mikes, H. M., \& Lewis, M. H. (2003). Selective blockade of spontaneous motor stereotypy via intrastriatalpharmacological manipulation. Pharmacology Biochemistry and Behavior, 74(4), 833-839. https://doi.org/10.1016/S0091-3057(02)01081-X

Quraishi, S., \& Frangou, S. (2002). N europsychology of bipolar disorder: a review *. In Journal of Affective Disorders

(Vol. 72). www.elsevier.com/locate/jad

Rung, J. P., Carlsson, A., Markinhuhta, K. R., \& Carlsson, M. L. (2005). The dopaminergic stabilizers (-)OSU6162 andACR16 reverse (+)-MK-801-induced social withdrawal in rats. Progress in Neuro-Psychopharmacology and Biological Psychiatry, 29(5), 833-839. https://doi.org/10.1016/j.pnpbp.2005.03.003

Sagvolden, T., Johansen, E. B., Aase, H., \& Russell, V. A. (2005). A dynamic developmental theory of attention- deficit/hyperactivity disorder (ADHD) predominantly hyperactive/impulsive and combined subtypes. $B e$ havioraland Brain Sciences, 28(3), 397-419. https://doi.org/10.1017/S0140525X05000075

Saha, S., Chant, D., Welham, J., \& McGrath, J. (2005). A systematic review of the prevalence of schizophrenia. In PLoSMedicine (Vol. 2, Issue 5, pp. 0413-0433). https://doi.org/10.1371/journal.pmed.0020141

Schultz, W., Dayan, P., \& Montague, P. R. (1997). A neural substrate of prediction and reward. Science, 275(5306),1593-1599. https://doi.org/10.1126/science.275.5306.1593

Shenton, M. E., Dickey, C. C., Frumin, M., Mccarley, R. W., \& Shenton, M. E. (2001). A review of MRI ®ndings inschizophrenia. www.elsevier.com/locate/schres

Tripp, G., \& Wickens, J. R. (2008). Research review: Dopamine transfer deficit: A neurobiological theory of altered reinforcement mechanisms in ADHD. In Journal of Child Psychology and Psychiatry and Allied Disciplines (Vol.49, Issue 7, pp. 691-704). https://doi.org/10.1111/j.1469-7610.2007.01851.x

Tune, L., Barta, P., Wong, D., Powers, R. E., Pearlson, G., Tien, A. Y., \& Wagner, H. N. (1996). Striatal dopamine D2 receptor quantification and superior temporal gyrus: Volume determination in 14 chronic schizophrenic subjects. InPsychiatry Research: Neuroimaging (Vol. 67).

Vilagut, G., Forero, C. G., Barbaglia, G., \& Alonso, J. (2016). Screening for depression in the general population with thecenter for epidemiologic studies depression (ces-d): A systematic review with meta-analysis. In PLOS ONE (Vol.

11, Issue 5). Public Library of Science. https://doi.org/10.1371/journal.pone.0155431 
Wang, D. V., \& Tsien, J. Z. (2011). Convergent processing of both positive and negative motivational signals by theVTA dopamine neuronal populations. PLoS ONE, 6(2). https://doi.org/10.1371/journal.pone.0017047

Xie, K., Kuang, H., \& Tsien, J. Z. (2013). Mild Blast Events Alter Anxiety, Memory, and Neural Activity Patterns in theAnterior Cingulate Cortex. PLoS ONE, 8(5). https://doi.org/10.1371/journal.pone.0064907

Yehuda, R., Flory, J. D., Southwick, S., \& Charney, D. S. (2006). Developing an agenda for translational studies of resilience and vulnerability following trauma exposure. Annals of the New York Academy of Sciences, $1071,379-$

396. https://doi.org/10.1196/annals.1364.028 